
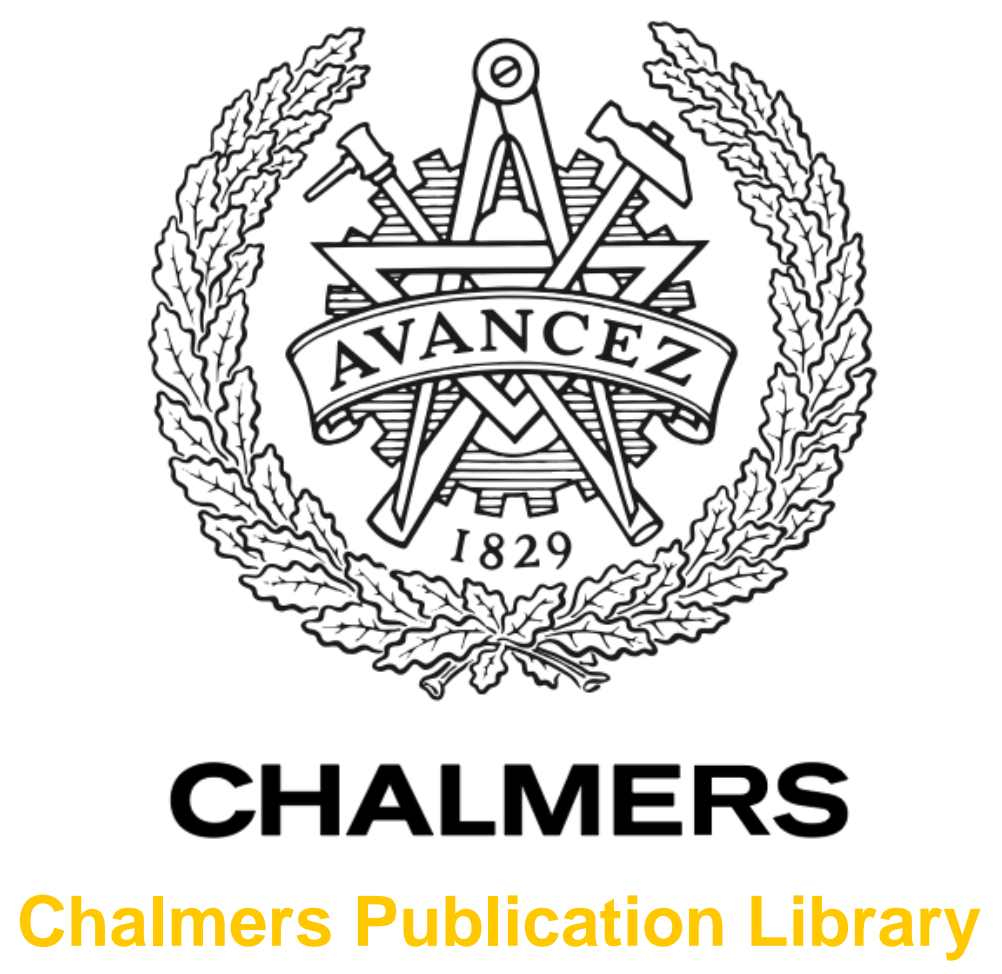

\title{
Underdamped Josephson junction as a switching current detector
}

This document has been downloaded from Chalmers Publication Library (CPL). It is the author's version of a work that was accepted for publication in:

\section{Applied Physics Letters (ISSN: 0003-6951)}

Citation for the published paper:

Oelsner, G. ; Revin, L. ; Il'Ichev, E. (2013) "Underdamped Josephson junction as a switching current detector". Applied Physics Letters, vol. 103(14),

http://dx.doi.org/10.1063/1.4824308

Downloaded from: http://publications.lib.chalmers.se/publication/186517

Notice: Changes introduced as a result of publishing processes such as copy-editing and formatting may not be reflected in this document. For a definitive version of this work, please refer to the published source. Please note that access to the published version might require a subscription. 


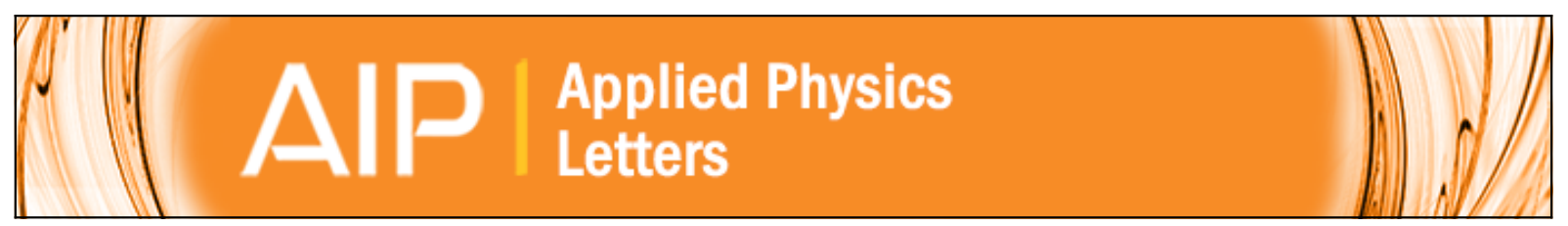

\section{Underdamped Josephson junction as a switching current detector}

G. Oelsner, L. S. Revin, E. Il'ichev, A. L. Pankratov, H.-G. Meyer, L. Grönberg, J. Hassel, and L. S. Kuzmin

Citation: Applied Physics Letters 103, 142605 (2013); doi: 10.1063/1.4824308

View online: http://dx.doi.org/10.1063/1.4824308

View Table of Contents: http://scitation.aip.org/content/aip/journal/apl/103/14?ver=pdfcov

Published by the AIP Publishing

$\stackrel{A}{A} \mathbb{P} P$ Re-register for Table of Content Alerts

Create a profile.

Sign up today! 


\title{
Underdamped Josephson junction as a switching current detector
}

\author{
G. Oelsner, ${ }^{1}$ L. S. Revin, ${ }^{2,3}$ E. Il'ichev,,${ }^{1,4}$ A. L. Pankratov, ${ }^{2,3, a)}$ H.-G. Meyer, ${ }^{1}$ L. Grönberg, ${ }^{5}$ \\ J. Hassel, ${ }^{5}$ and L. S. Kuzmin ${ }^{3,6}$ \\ ${ }^{1}$ Institute of Photonic Technology IPHT, D-07702 Jena, Germany \\ ${ }^{2}$ Institute for Physics of Microstructures of RAS, GSP-105, Nizhny Novgorod 603950, Russia \\ ${ }^{3}$ Laboratory of Cryogenic Nanoelectronics, Nizhny Novgorod State Technical University, \\ Nizhny Novgorod, Russia \\ ${ }^{4}$ Novosibirsk State Technical University, 20 Karl Marx Avenue, 630092 Novosibirsk, Russia \\ ${ }^{5}$ VTT Technical Research Center of Finland, P.O. Box 1000, FI-02044 VTT, Finland \\ ${ }^{6}$ Chalmers University of Technology, SE-41296 Gothenburg, Sweden
}

(Received 6 July 2013; accepted 20 September 2013; published online 3 October 2013)

\begin{abstract}
We demonstrate the narrow switching distribution of an underdamped Josephson junction from the zero to the finite voltage state at millikelvin temperatures. We argue that such junctions can be used as ultrasensitive detectors of the single photons in the $\mathrm{GHz}$ range, operating close to the quantum limit: a given initial (zero voltage) state can be driven by an incoming signal to the finite voltage state. The width of the switching distribution at a nominal temperature of about $T=10 \mathrm{mK}$ was $4.5 \mathrm{nA}$, which corresponds to an effective noise temperature of the device below 60 mK. (C) 2013 AIP Publishing LLC. [http://dx.doi.org/10.1063/1.4824308]
\end{abstract}

Nowadays ultrasensitive sensors, based on superconducting materials, are widely used in the various fields of science and applications, including the detection of electromagnetic signals close to the quantum limit. ${ }^{1}$ In particular, circuit quantum electrodynamic components, ${ }^{2}$ which apply for microwave quantum engineering, require such type of detectors for information processing devices. ${ }^{3}$ It is quite natural to optimize switching properties of a current-biased Josephson junction ${ }^{4}$ (CBJJ) and utilize it as a microwave detector. In this letter we demonstrate similar detector with a noise temperature below $60 \mathrm{mK}$.

The CBJJ is a device in which the Josephson phase variable is trapped in a washboard potential. Modulation of the potential tilt or a radiation field can lead to an escape of the "particle" from the well, which corresponds to a voltage drop over the CBJJ., This switching can also occur due to thermal activation (TA) $)^{7-9}$ and due to macroscopic quantum tunneling (MQT). ${ }^{10}$

Quantitatively, the dynamics of a CBJJ can be described by a fictitious phase particle of mass $M=C\left(\Phi_{0} / 2 \pi\right)^{2}$ and damping coefficient $1 / R C$ moving along the generalized coordinate $\varphi$ in a washboard potential

$$
U(i, \phi)=-E_{J}(i \phi+\cos \phi),
$$

where $R$ and $C$ are the effective shunt resistance and capacitance of the junction, respectively, $\Phi_{0}$ is the magnetic flux quantum, $E_{J}=I_{c} \Phi_{0} / 2 \pi$ is the characteristic scale of the Josephson energy, and $i=I / I_{c}$ is the normalized bias current. If thermal and quantum fluctuations are absent and the bias current is smaller than the critical one $i<1$, the junction is in the zero-voltage state, so the corresponding phase particle is located in the potential well with the barrier height given by

$$
\Delta U(i)=2 E_{J}\left[\sqrt{1-i^{2}}-i \arccos (i)\right]
$$

\footnotetext{
${ }^{a)}$ Electronic mail: alp@ipmras.ru
}

and the oscillation frequency of the particle at the bottom of the well

$$
\omega_{0}=\sqrt{\frac{\partial^{2} U / \partial^{2} \phi}{M}}=\omega_{p}\left(1-i^{2}\right)^{1 / 4},
$$

where $\omega_{p}=\sqrt{2 \pi I_{c} / \Phi_{0} C}$ is the plasma frequency. For $i<1$ and finite temperatures $T>0$, the particle may escape from the potential well either by thermal activation or by macroscopic quantum tunneling, resulting in the junction's switching from the zero to the finite voltage state. At high temperatures the escape of the particle from the well is mainly due to thermal activation processes, and its rate can be found from Kramers formula ${ }^{11,12}$

$$
\Gamma_{t}=\frac{\omega_{0}}{2 \pi} a_{t} \exp \left(-\frac{\Delta U}{k_{B} T}\right),
$$

where $a_{t}=4 /\left(\sqrt{1+Q k_{B} T / 1.8 \Delta U}+1\right)^{2}$ is a temperature and damping dependent thermal prefactor, and $Q=\omega_{0} R C$ is the quality factor. For even larger temperatures or for bias currents approaching the critical current, when the condition $\Delta U \gg k_{B} T$ is not fulfilled and the Kramers formula can not be applied, one can resort to a more general expression. ${ }^{13}$ For smaller temperatures $T$, the quantum tunneling becomes dominating over thermal activation. In the MQT regime the escape rate is given by ${ }^{14,15}$

$$
\Gamma_{q}=\frac{\omega_{0}}{2 \pi} \sqrt{\frac{B}{2 \pi}} \exp (-B),
$$

with $B=\frac{\Delta U}{\hbar \omega_{0}}[7.2+8 A / Q]$. The second term in $B$ represents the dissipative corrections, where $A$ is a numerical factor. These corrections are particularly important for low-currentdensity Josephson junctions. ${ }^{15,16}$

The temperature at which the system passes from the MQT to the TA regime is called the crossover temperature $^{17}$ 


$$
T_{c r}=\frac{\hbar \omega_{0}}{2 \pi k_{B}}\left[\sqrt{1+\left(\frac{1.2 A}{2 Q}\right)^{2}}-\frac{1.2 A}{2 Q}\right] .
$$

For a Josephson junction this temperature is equivalent to the noise temperature ${ }^{18}$ of a switching current detector. Note, additionally, that increase of dissipation lowers $T_{c r}$ in Eq. (6) and diminishes quantum tunneling effects in Eq. (5) ( $T_{c r}$ is the decaying function of $1.2 A / 2 Q$ ).

In the adiabatic approximation (see, e.g., Ref. 19), i.e., when the bias current variation is slow in comparison with characteristic time scales of the system, the probability for the particle to escape from the well and therefore to generate a voltage drop for current $i(t)$ is

$$
P(i)=\exp \left[-\frac{1}{\partial i / \partial t} \int_{0}^{i} \Gamma\left(i^{\prime}\right) d i^{\prime}\right] .
$$

The escape of the Josephson tunnel junction phase is investigated experimentally by measuring its switching statistics from the zero-voltage state to the finite voltage state. The so-called switching current probability distribution $W(I)=\frac{\partial P(i)}{\partial i} \frac{1}{I_{c}}$ is found by averaging over a large number of measurements of the switching current $I_{S W}$ and generating a histogram.

The sample, a current-biased Josephson junction, was fabricated using the $30 \mathrm{~A} / \mathrm{cm}^{2}$ process at VTT, Finnland. ${ }^{20}$ A small current density was chosen to reduce the heating power connected to switching of the junction into the voltage state and therewith heating of the sample. The junction consists of a $\mathrm{NbAlO}_{\mathrm{x}} \mathrm{Nb}$ trilayer and has a nominal capacitance of $C \approx 0.33 \mathrm{pF}$ and a total damping resistance of $R \approx 0.44 \mathrm{k} \Omega$. The sample was thermally anchored to the base of a dilution refrigerator, providing a minimal temperature below $10 \mathrm{mK}$ and enclosed by both, a mu-metal and superconducting shielding. Further penetration of noise to the sample was avoided by the use of filtered twisted-pair lines in a four point measurement design for the connection of the junction to the measurement devices. An $R C$-filter with cut-off frequency of $10 \mathrm{kHz}$ at the $4 \mathrm{~K}$ stage together with a $L C$-filter with similar cut-off and a copper-powder filter both at base temperature stage were used. The switching current distributions of the sample were measured at bath temperatures $T$ between $10 \mathrm{mK}$ and $1000 \mathrm{mK}$. The current was ramped up by the following law: it is set in steps of $\Delta I=0.1 \mathrm{nA} / \mathrm{ms}$ during $10 \mathrm{~ms}$ with a waiting time of $10 \mathrm{~ms}$ between steps.

In Fig. 1 the temperature dependence of the mean $\left\langle I_{S W}\right\rangle$ and the standard deviation $\sigma$ of the switching current $I_{S W}$ (symbols), together with the results calculated from the TA and MQT theories (curves) are shown. In the temperature range between $1 \mathrm{~K}$ and approximately $56 \mathrm{mK} \sigma$ decreases with $T$ indicating that TA is the dominant escape mechanism. In this temperature range, $W(I)$ depends weakly on $C$ and $R$, so the critical current $I_{c}$ can be determined by fitting $W(I)$ using the TA theory. In our case the $I_{c}$ has a value of $2.2 \mu \mathrm{A}$. One can see that below the crossover temperature, $\sigma$ demonstrates saturation at the level $\sim 4.5 \mathrm{nA}$, meaning that the quantum tunneling through the barrier is the main mechanism of escape in this case. ${ }^{21-24}$ The numerical factor

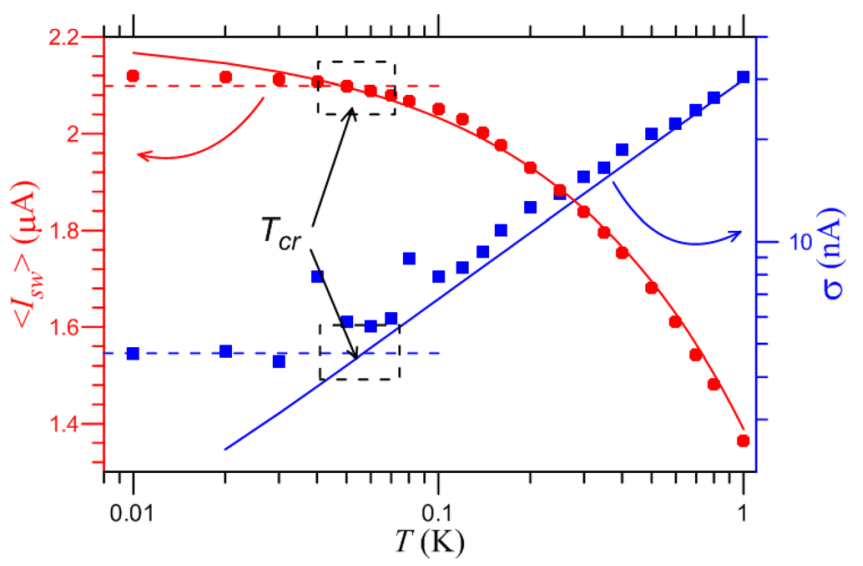

FIG. 1. The standard deviation $\sigma$ (rectangular symbols) and the mean value $\left\langle I_{S W}\right\rangle$ (circular symbols) of the $W(I)$ distribution vs. the nominal temperature $T$ of the mixing chamber of the dilution fridge. The solid curves mark the predictions from TA theory. Dashed lines-from MQT theory. The experimental MQT-to-TA crossover temperature $T_{c r}$ around $56 \mathrm{mK}$ is indicated.

$A$ can be determined using $W(I)$ at this regime, and an agreement to the data was found for $A \approx 10$. Taking the $W(I)$ peak position in the MQT regime, we find that the crossover temperature calculated from these parameters is roughly $T_{c r} \approx 56 \mathrm{mK}$.

Interestingly, the same number can be obtained by making use of the simplified consideration. Suppose that the current (and, therefore, the Josephson junction energy) fluctuations are caused by the thermal noise $k T .^{25}$ This way, the switching event occurs at the junction phase $\varphi$ in the vicinity of $\pi / 2$ so that $\Delta \phi=\pi / 2-\phi \ll 1$. Therefore, the Josephson energy close to the switching point $\left(\Phi_{0} I_{c} / 2 \pi\right) \cos \phi$ can be written as $\left(\Phi_{0} I_{c} \sin \Delta \phi\right) / 2 \pi$. Taking into account $\Delta \phi>0$ we obtain that $\Delta I=I_{C} \sin \Delta \phi$ is half of the width of the experimentally obtained switching current distribution. Thus, the noise temperature or $T_{c r}$ can be reconstructed from $k T_{c r}=\left(\Phi_{0} \Delta I\right) / 2 \pi$ and $T_{c r} \simeq 54 \mathrm{mK}$.

Let us now consider possible ways to reduce the crossover temperature. In Fig. 2 its theoretically obtained value is presented versus the current rate for different values of the capacitance $C$. For the underdamped Josephson junction the increase of the bias current rate $\dot{I}$ leads to an approach of the mean switching current $\left\langle I_{S W}\right\rangle$ to the critical current $I_{c}$.

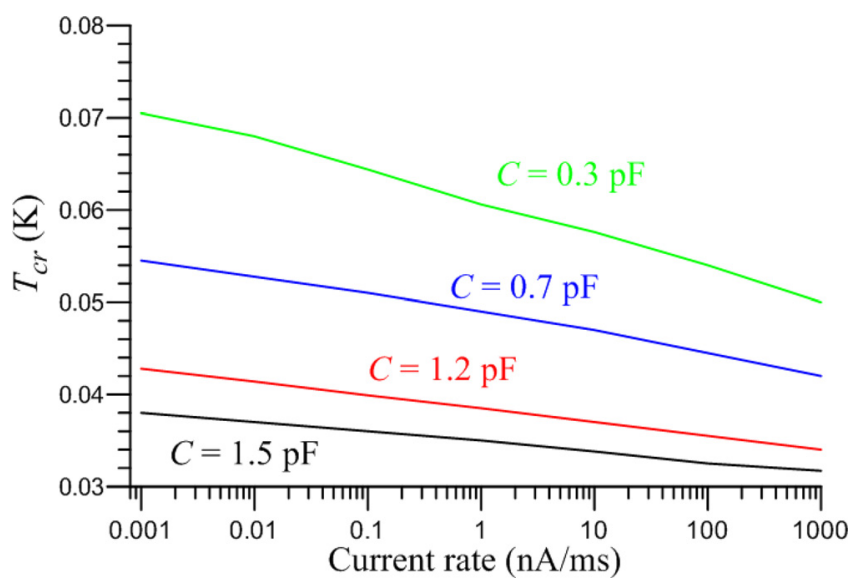

FIG. 2. The crossover temperature $T_{c r}$ vs current rate $\dot{I}$ for different values of junction capacitance $C$. 
The rapid current growth does not allow thermal fluctuations to shake the system and to switch it to the finite voltage state. $^{26,27}$ Similar picture arises in the MQT regime as well. The dependence $T_{c r}(\dot{I})$ is logarithmic with different slope for different $C$ values. Thus, the increase in the rate $\dot{I}$ and accordingly the increase of $\left\langle I_{S W}\right\rangle$ leads to a decrease of the frequency $\omega_{0}$ (Eq. (3)) and the crossover temperature $T_{c r}$ (Eq. (6)), see Fig. 2. On one hand the crossover temperature decreases with increasing in capacitance by an exponential law. On the other hand the changing of $C$ leads to changing of $\omega_{0}$ by a square root dependence. In such a way for creation of a high sensitive detector the parameters reducing $T_{c r}$ should be selected according to the operating frequency range.

In summary, the switching current distribution measurements presented here demonstrate a relatively low temperature crossover $T_{c r} \approx 56 \mathrm{mK}$. It is important to note that this result was obtained for a low critical current and a slow current rate. The given device has important applications in the context of supersensitive detection. For example, the ambitious task of implementation of a single-photon detector in the $\mathrm{GHz}$ range has certain perspectives. Indeed, the amplitude of the current which corresponds to a single-photon at the frequency of $2.5 \mathrm{GHz}$ can be estimated as $I=\sqrt{h \nu / L_{R}}$, where $L_{R}$ is the inductance of the corresponding part of the transmission line. Since usually such parts are $\lambda / 2$ (Ref. 28) or $\lambda / 4$ (Ref. 29) waveguide resonators, corresponding estimations yield $12 \mathrm{nA}$ and $18 \mathrm{nA}$, respectively. These numbers are quite optimistic since half of the switching current distribution of $2.2 \mathrm{nA}$ was realized. However, apart from adiabatic regime, here dynamical properties of this quantum mechanical system should be analysed, and first step has already been done. ${ }^{4}$ Additionally, such detectors can be used in more complex superconducting schemes, where the information is transferred by magnetic flux quanta moving along in a similar transmission line. ${ }^{30,31}$ Any external influence is changing the flux dynamics, whether it is an external magnetic field or a superconducting qubit state, is registered by the readout device. It is important to notice that in this case the quantum non-destructive measurements, which play an important role in applications, can be realized.

The research leading to these results was received funding from the European Community's Seventh Framework Programme (FP7/2007-2013) under Grant No. 270843 (iQIT). The work was also supported by Russian Ministry of
Science (Grant No. 11.G34.31.0029) and Dynasty Foundation.

${ }^{1}$ S. Anders, M. G. Blamire, F.-Im. Buchholz, D.-G. Crété, R. Cristiano, P. Febvre, L. Fritzsch, A. Herr, E. Il'ichev, J. Kohlmann et al., Physica C 470, 2079 (2010).

${ }^{2}$ A. Wallraff, D. I. Schuster, A. Blais, L. Frunzio, R.-S. Huang, J. Majer, S. Kumar, S. M. Girvin, and R. J. Schoelkopf, Nature 431, 162 (2004).

${ }^{3}$ V. Zakosarenko, N. Bondarenko, S. H. W. van der Ploeg, A. Izmalkov, S. Linzen, J. Kunert, M. Grajcar, E. Il'ichev, and H.-G. Meyer, Appl. Phys. Lett. 90, 022501 (2007).

${ }^{4}$ C. K. Andersen and K. Mölmer, Phys. Rev. A 87, 052119 (2013).

${ }^{5}$ A. Wallraff, A. Lukashenko, C. Coqui, A. Kemp, T. Duty, and A. V. Ustinov, Rev. Sci. Instrum. 74, 3740 (2003).

${ }^{6}$ H. F. Yu, X. B. Zhu, Z. H. Peng, W. H. Cao, D. J. Cui, Ye Tian, G. H. Chen, D. N. Zheng, X. N. Jing, Li Lu et al., Phys. Rev. B 81, 144518 (2010).

${ }^{7}$ J. M. Martinis and R. L. Kautz, Phys. Rev. Lett. 63, 1507 (1989).

${ }^{8}$ M. Castellano, R. Leoni, G. Torrioli, F. Chiarello, C. Cosmelli, A. Costantini, G. Diambrini-Palazzi, P. Carelli, R. Cristiano, and L. Frunzio, J. Appl. Phys. 80, 2922 (1996).

${ }^{9}$ J. M. Martinis, M. H. Devoret, and J. Clarke, Phys. Rev. B 35, 4682 (1987).

${ }^{10}$ M. H. Devoret, J. M. Martinis, and J. Clarke, Phys. Rev. Lett. 55, 1908 (1985).

${ }^{11}$ H. A. Kramers, Physica (Utrecht) 7, 284 (1940).

${ }^{12}$ P. Hanggi, P. Talkner, and M. Borkovec, Rev. Mod. Phys. 62, 251 (1990).

${ }^{13}$ A. N. Malakhov and A. L. Pankratov, Physica C 269, 46 (1996).

${ }^{14}$ A. O. Caldeira and A. J. Leggett, Phys. Rev. Lett. 46, 211 (1981).

${ }^{15}$ F. V. Richard and A. W. Richard, Phys. Rev. Lett. 47, 265 (1981).

${ }^{16}$ J. M. Martinis and H. Grabert, Phys. Rev. B 38, 2371 (1988).

${ }^{17}$ H. Grabert and U. Weiss, Phys. Rev. Lett. 53, 1787 (1984).

${ }^{18}$ P. Silvestrini, O. Liengme, and K. E. Gray, Phys. Rev. B 37, 1525 (1988).

${ }^{19}$ A. L. Pankratov and M. Salerno, Phys. Lett. A 273, 162 (2000).

${ }^{20}$ M. G. Castellano, L. Grönberg, P. Carelli, F. Chiarello, C. Cosmelli, R. Leoni, S. Poletto, G. Torrioli, J. Hassel, and P. Helistö, Supercond. Sci. Technol. 19, 860 (2006)

${ }^{21}$ S. Washburn, R. A. Webb, R. F. Voss, and S. M. Faris, Phys. Rev. Lett. 54, 2712 (1985).

${ }^{22}$ A. Wallraff, A. Lukashenko, J. Lisenfeld, A. Kemp, M. V. Fistul, Y. Koval, and A. V. Ustinov, Nature 425, 155 (2003).

${ }^{23}$ M. V. Fistul, A. Wallraff, Y. Koval, A. Lukashenko, B. A. Malomed, and A. V. Ustinov, Phys. Rev. Lett. 91, 257004 (2003).

${ }^{24}$ R. Fazio and H. van der Zant, Phys. Rep. 355, 235 (2001).

${ }^{25}$ K. K. Likharev, Dynamics of Josephson Junctions and Circuits (Gordon and Breach, New York, 1986).

${ }^{26}$ A. L. Pankratov and B. Spagnolo, Phys. Rev. Lett. 93, 177001 (2004).

${ }^{27}$ A. V. Gordeeva and A. L. Pankratov, Appl. Phys. Lett. 88, 022505 (2006).

${ }^{28}$ G. Oelsner, S. H. W. van der Ploeg, P. Macha, U. Hubner, D. Born, S. Anders, E. Il'ichev, H.-G. Meyer, M. Grajcar, S. Wünsch et al., Phys. Rev. B 81, 172505 (2010).

${ }^{29}$ M. Jerger, S. Poletto, P. Macha, U. Hubner, E. Il'ichev, and A. V. Ustinov, Appl. Phys. Lett. 101, 042604 (2012).

${ }^{30}$ A. Herr, A. Fedorov, A. Shnirman, E. Il'ichev, and G. Schön, Supercond. Sci. Technol. 20, S450 (2007).

${ }^{31}$ A. L. Pankratov, A. V. Gordeeva, and L. S. Kuzmin, Phys. Rev. Lett. 109, 087003 (2012). 